\title{
THE CONSTRUCTION OF ALE SPACES AS HYPER-KÄHLER QUOTIENTS
}

\author{
P. B. KRONHEIMER
}

\section{Introduction}

According to the definition given by Calabi [4], a Riemannian manifold $(X, g)$ is hyper-Kähler if it is equipped with three automorphisms $I, J, K$ of the tangent bundle which satisfy the relations of the quaternion algebra $\mathbf{H}$ and are covariant constant with respect to the Levi-Civita connection:

$$
I^{2}=J^{2}=K^{2}=-1, \quad I J=-J I=K, \quad \nabla I=\nabla J=\nabla K=0 .
$$

These conditions imply in particular that each of $I, J$ and $K$ defines an integrable complex structure on $X$ and that the metric $g$ is Kähler with respect to all three; the three Kähler forms $\omega_{1}, \omega_{2}, \omega_{3}$ are therefore closed, giving three symplectic structures to $X$. In dimension 4 , a simply-connected Riemannian manifold admits such a hyper-Kähler structure precisely when the Riemann curvature tensor is either self-dual or anti-self-dual. A complete, hyper-Kähler 4-manifold is therefore a self-dual, positive-definite solution to Einstein's equations in vacuum (a self-dual gravitational instanton), and it is with examples of such manifolds that we are concerned.

This paper describes the construction of a particular family of hyper-Kähler 4-manifolds, the so-called ALE spaces [6]. ALE stands for asymptotically locally Euclidean and describes a Riemannian 4-manifold with just one end which at infinity resembles a quotient $\mathbf{R}^{4} / \Gamma$ of Euclidean space $\mathbf{R}^{4}$ by a finite group $\Gamma$ of identifications. The Riemannian metric $g$ is required to approximate the Euclidean metric up to $O\left(r^{-4}\right)$,

$$
g^{i j}=\delta^{i j}+O\left(r^{-4}\right)
$$

with appropriate decay in the derivatives of $g^{i j}$. A large class of such ALE spaces was discovered by Gibbons and Hawking [7]. For each integer $k \geq 2$, they constructed a family of spaces, depending on $3 k-6$ parameters, which had self-dual curvature and resembled at infinity a quotient of $\mathbf{R}^{4}$ by a cyclic group $\Gamma$ of order $k$. These 'multi-Eguchi-Hanson' metrics were obtained also by Hitchin [8], who derived them by an application of Penrose's nonlinear

Received June 12, 1987 and, in revised form, January 29, 1988. 
graviton construction. Hitchin's approach pointed to a close relationship with the deformation theory of the complex quotient singularities $\mathbf{C}^{2} / \Gamma$ and strongly suggested the existence of other families of ALE gravitational instantons associated with the other finite subgroups $\Gamma \subset \mathrm{SU}(2)$ - the binary dihedral, tetrahedral, octahedral and icosahedral groups. These conjectured ALE spaces should be similarly related to the quotient singularities $\mathbf{C}^{2} / \Gamma$, the so-called Kleinian singularities, or rational double points. The construction we describe confirms this conjecture.

The following theorem (our main result) has been announced in [12]. Let $\Gamma$ be a finite subgroup of $\mathrm{SU}(2)$, let $\widetilde{\mathrm{C}^{2} / \Gamma} \rightarrow \mathrm{C}^{2} / \Gamma$ be the minimal resolution of the quotient singularity, and let $X$ be the smooth 4-manifold which underlies the complex surface $\mathbf{C}^{2} / \Gamma$.

Theorem 1.1. Let three coholomogy classes $\alpha_{1}, \alpha_{2}, \alpha_{3} \in H^{2}(X ; \mathbf{R})$ be given which satisfy the nondegeneracy condition

$$
\begin{aligned}
& \text { for each } \Sigma \in H_{2}(X ; \mathbf{Z}) \text { with } \Sigma \cdot \Sigma=-2 \text {, there exists } \\
& i \in\{1,2,3\} \text { with } \alpha_{i}(\Sigma) \neq 0 .
\end{aligned}
$$

Then there exists on $X$ an ALE hyper-Kähler structure for which the cohomology classes of the Kähler forms $\left[\omega_{i}\right]$ are the given $\alpha_{i}$.

The proof of this result is a direct application of a procedure which is already known to produce a wide variety of hyper-Kähler manifolds, including the multi-Eguchi-Hanson spaces. This is the hyper-Kähler quotient construction of Hitchin et al. [9], a modification of the symplectic quotient, or reduced phase space, familiar in symplectic geometry. We review this construction in $\S 2$ and then apply it in a particular case to produce a family of hyper-Kähler 4-manifolds. In $\S 3$ we show that these manifolds are diffeomorphic to $\widetilde{C^{2} / \Gamma}$ and that their metrics are ALE. The proof of Theorem 1.1 is completed in $\S 4$ where we calculate the cohomology classes of the Kähler forms on each member of the family.

In a later paper [13] we shall show that the construction presented here is complete: every ALE hyper-Kähler 4-manifold (and therefore every simplyconnected, ALE solution to the self-dual Einstein equations) is isometric to a member of one of the families produced in $\S 2$. These results, obtained by twistor methods, may be summarized as follows.

Theorem 1.2. Every ALE hyper-Kähler 4-manifold is diffeomorphic to the minimal resolution of $\mathrm{C}^{2} / \Gamma$ for some $\Gamma \subset \mathrm{SU}(2)$, and the cohomology classes of the Kähler forms on such a manifold must satisfy condition (*). 
Theorem 1.3. If $X_{1}$ and $X_{2}$ are two ALE hyper-Kähler 4-manifolds, and there is a diffeomorphism $X_{1} \rightarrow X_{2}$ under which the cohomology classes of the Kähler forms agree, then $X_{1}$ and $X_{2}$ are isometric.

\section{A family of hyper-Kähler manifolds}

We now review the Kähler and hyper-Kähler quotient constructions. Let $M$ be a simply-connected Kähler manifold, and $F$ a compact Lie group acting on $M$ so as to preserve the metric $g$ and the complex structure $I: T M \rightarrow T M$. Let $\mathfrak{f}$ be the Lie algebra of $F$, and for each $\xi \in \mathfrak{f}$ let $V_{\xi}$ be the vector field on $M$ which the action of $\xi$ generates. According to the familiar definition from symplectic geometry, a moment map for the action of $F$ on $M$ is an $F$-equivariant map

$$
\mu: M \rightarrow f^{*}
$$

with the property that, for each $\xi \in \mathfrak{f}$, the function $\mu \cdot \xi: M \rightarrow \mathbf{R}$ satisfies

$$
\operatorname{grad}(\mu \cdot \xi)=I\left(V_{\xi}\right)
$$

Under our assumption that $M$ is simply-connected, a moment map always exists and is unique to within the addition of a constant $\varsigma \in Z \subset f^{*}$, where $Z$ is the space of $F$-invariant elements essentially the dual of the centre of $\mathfrak{f}$. If $\mu$ is a moment map and $\varsigma \in Z$, then $\mu^{-1}(\varsigma) \subset M$ is invariant under $F$. The quotient space $X=\mu^{-1}(\varsigma) / F$ is the Kähler quotient of $M$ by $F$. Note that if the center of $F$ is nontrivial, then the Kähler quotient is not unique, for an element $\zeta \in Z$ must be chosen.

Now suppose that $M$ is hyper-Kähler and that $F$ acts so as to preserve $g$ as well as all three complex structures. There are then three moment maps (one for each of $I, J$ and $K$ ) which one puts together to form the hyper-Kähler moment map

$$
\mu=\left(\mu_{1}, \mu_{2}, \mu_{3}\right): M \rightarrow \mathbf{R}^{3} \otimes f^{*} .
$$

Following Hitchin et al. [9], after choosing $\varsigma \in \mathbf{R}^{3} \otimes Z$, one defines the hyperKähler quotient as

$$
X=\mu^{-1}(\varsigma) / F
$$

The following proposition gives the properties of Kähler (resp. hyper-Kähler) quotients which are proved in [9].

Proposition 2.1. Suppose that $F$ acts freely on $\mu^{-1}(\zeta)$. Then

(i) $d \mu$ has full rank at all points of $\mu^{-1}(\zeta)$, so that $X$ is a nonsingular manifold of dimension $\operatorname{dim} M-2 \operatorname{dim} F($ resp. $\operatorname{dim} M-4 \operatorname{dim} F)$,

(ii) the metric $g$ and complex structures $I$ (resp. $I, J, K$ ) descend to $X$, and equipped with these, $X$ is Kähler (resp. hyper-Kähler). 
We make a particular application of this hyper-Kähler quotient construction. Let $\Gamma$ be a finite subgroup of $\mathrm{SU}(2)$, let $R$ be its regular representation and $Q$ its canonical 2-dimensional representation, and put

$$
P=Q \otimes \operatorname{End}(R) \text {. }
$$

Define $M=P^{\Gamma}$, the space of $\Gamma$-invariant elements in $P$. We make $P$ and $M$ into right modules over $\mathbf{H}$ as follows. First, we regard $Q$ as a rank-1 $\mathbf{H}$ module in such a way that $\mathrm{SU}(2)$ coincides with the symplectic group $\mathrm{Sp}(Q)$ of $\mathbf{H}$-linear isometries of $Q$. Next, a choice of invariant hermitian metric on $R$ gives $\operatorname{End}(R)$ a real structure, the antilinear involution $\alpha \mapsto \alpha^{*}$. As the tensor product of an $\mathbf{H}$-module and a real space, $P$ then inherits an $\mathbf{H}$ module structure. Explicitly, if we choose an orthonormal basis for $Q$ so as to represent an element of $P$ as a pair of endomorphisms $(\alpha, \beta)$, the action of $J$ is given by

$$
J(\alpha, \beta)=\left(-\beta^{*}, \alpha^{*}\right), \quad \alpha, \beta \in \operatorname{End}(R) .
$$

The action of $\Gamma$ on $P$ is $\mathbf{H}$-linear and the subspace $M$ is therefore an $\mathbf{H}$ submodule. Explicitly again, a pair $(\alpha, \beta)$ lies in $M$ if it satisfies the condition that, for each $\gamma=\left(\begin{array}{cc}u & v \\ -\bar{v} & \bar{u}\end{array}\right) \in \Gamma$ we have

$$
R\left(\gamma^{-1}\right) \alpha R(\gamma)=u \alpha+v \beta, \quad R\left(\gamma^{-1}\right) \beta R(\gamma)=-\bar{v} \alpha+\bar{u} \beta
$$

Identifying each tangent space to $M$ with $M$ itself, we regard this linear space as a flat hyper-Kähler manifold.

Let $\mathrm{U}(R)$ be the group of unitary transformations of $R$ and let $F \subset \mathrm{U}(R)$ be the subgroup consisting of those elements which commute with the action of $\Gamma$ on $R$. The natural action of $F$ on $P$ given by

$$
(\alpha, \beta) \mapsto\left(f \alpha f^{-1}, f \beta f^{-1}\right), \quad f \in F,
$$

is $\mathbf{H}$-linear and preserves the subspace $M$. As the circle subgroup $T$ of scalars acts trivially, we therefore have an action of $F / T$ on $M$ which preserves $I, J$, and $K$.

The moment map for this action is easily written down: if one identifies $(\mathfrak{f} / \mathfrak{t})^{*}$ with the traceless elements of $f \subset \operatorname{End}(R)$, then the three components of $\mu$ are given by

$$
\begin{aligned}
& \mu_{1}(\alpha, \beta)=\frac{1}{2} i\left(\left[\alpha, \alpha^{*}\right]+\left[\beta, \beta^{*}\right]\right), \\
& \mu_{2}(\alpha, \beta)=\frac{1}{2}\left([\alpha, \beta]+\left[\alpha^{*}, \beta^{*}\right]\right), \\
& \mu_{3}(\alpha, \beta)=\frac{1}{2} i\left(-[\alpha, \beta]+\left[\alpha^{*}, \beta^{*}\right]\right) .
\end{aligned}
$$

We have picked out the preferred moment map which vanishes at the origin. Applying the quotient construction, we choose a triple $\varsigma=\left(\varsigma_{1}, \varsigma_{2}, \varsigma_{3}\right) \in \mathbf{R}^{3} \otimes Z$, where $Z \subset(\mathfrak{f} / \mathfrak{t})^{*}$ is the center, and set

$$
X_{\varsigma}=\mu^{-1}(\varsigma) / F \text {. }
$$


Our claim is that, as $\zeta$ varies, we obtain in the family of spaces $X_{\varsigma}$ all the ALE spaces whose existence is asserted by Theorem 1.1.

In crder to give a different description of the space $M$ and the group $F$, a short digression is necessary. Let $R_{0}, R_{1}, \cdots, R_{r}$ be the irreducible representation of $\Gamma$ with $R_{0}$ the trivial representation, let $Q$ be the 2-dimensional representation as before, and let

$$
Q \otimes R_{i}=\bigoplus_{j} a_{i j} R_{j}
$$

be the decomposition of $Q \otimes \mathbf{R}_{i}$ into irreducibles. McKay [15] observed that the matrix $A=\left(a_{i j}\right)$, whose entries are all either 0 or 1 , is the adjacency matrix of a simply-laced extended Dynkin diagram $\bar{\Delta}(\Gamma)$; equivalently, $\bar{C}=2 I-A$ is an extended Cartan matrix. The trivial representation $R_{0}$ corresponds to the extra vertex of the extended diagram, and the representations $R_{1}, \cdots, R_{r}$ therefore correspond to a set of simple roots $\theta_{1}, \cdots, \theta_{r}$ for the associated root system. We write $\theta_{0}$ for the negative of the highest root and note that, as McKay further observed, if

$$
\theta_{0}=-\sum_{1}^{r} n_{i} \theta_{i}
$$

is the expression for $\theta_{0}$ in terms of the simple roots, then the coefficient $n_{i}$ is precisely the dimension of $R_{i}$. The assignment of $\bar{\Delta}(\Gamma)$ to $\Gamma$ sets up a one-to-one correspondence between the finite subgroups of $\mathrm{SU}(2)$ and the simply-laced diagrams $A_{r}, D_{r}, E_{6}, E_{7}$ and $E_{8}$.

The regular representation of $\Gamma$ decomposes as

$$
R=\bigoplus_{i} \mathrm{C}^{n_{i}} \otimes R_{i}
$$

Accordingly, $M$ may be written

$$
\begin{aligned}
M & =\operatorname{Hom}_{\Gamma}(R, Q \otimes R) \\
& =\bigoplus_{i, j} \operatorname{Hom}_{\Gamma}\left(R_{i}, Q \otimes R_{j}\right) \otimes \operatorname{Hom}\left(\mathbf{C}^{n_{i}}, \mathbf{C}^{n_{j}}\right) \\
& =\bigoplus_{i, j} a_{i j} \operatorname{Hom}\left(\mathbf{C}^{n_{i}}, \mathbf{C}^{n_{j}}\right)
\end{aligned}
$$

and, by McKay's observation, this description may be rephrased as

$$
M=\bigoplus_{i \rightarrow j} \operatorname{Hom}\left(\mathbf{C}^{n_{i}}, \mathbf{C}^{n_{j}}\right),
$$

where the sum is taken over all edges of $\bar{\Delta}(\Gamma)$, and each edge appears twice in the sum, once with each orientation. The group $F$ can be similarly described 
in terms of $\bar{\Delta}(\Gamma)$ : it is a product of unitary groups

$$
F=\underset{i}{\times} \mathrm{U}\left(n_{i}\right)
$$

with one factor for each vertex of $\bar{\Delta}(\Gamma)$, and it acts on $M$ in the obvious way. Using these descriptions, we compute

$$
\begin{aligned}
\operatorname{dim}_{\mathbf{R}} M & =\sum_{i, j} 2 a_{i j} n_{i} n_{j}=\sum_{i, j}\left(4 \delta_{i j}-2 c_{i j}\right) n_{i} n_{j} \\
& =\sum_{i} 4 n_{i}^{2}=4|\Gamma| \\
& \operatorname{dim}_{\mathbf{R}} F=\sum_{i} n_{i}^{2}=|\Gamma| .
\end{aligned}
$$

The center of the Lie algebra $f$ is spanned by the elements $\sqrt{-1} \pi_{i}$, where $\pi_{i}$ is the projection $\pi_{i}: R \rightarrow \mathbf{C}^{n_{i}} \otimes R_{i}(i=0, \cdots, r)$. Writing $h$ for the real Cartan algebra associated to the Dynkin diagram, we define a linear map $\rho$ from the center of $f$ to $h^{*}$ by

$$
\rho: \sqrt{-1} \pi_{i} \mapsto n_{i} \theta_{i} .
$$

The kernel of $\rho$ is the one-dimensional subalgebra $t \subset \mathfrak{f}$, so that on the dual spaces, $\rho$ induces an isomorphism

$$
\tau: Z \rightarrow h .
$$

For each root $\theta$ (not necessarily simple), we write $D_{\theta}=\operatorname{Ker}(\theta \circ \tau) \subset Z$. Thus we identify $Z$ with the Cartan algebra, and the hyperplanes $D_{\theta}$ are the walls of the Weyl chambers.

Proposition 2.8. If $F / T$ does not act freely on $\mu^{-1}(\zeta)$, then $\zeta$ lies in one of the codimesion-3 subspaces $\mathbf{R}^{3} \otimes D_{\theta} \subset \mathbf{R}^{3} \otimes Z$, where $\theta$ is a root.

Proof. Suppose that $(\alpha, \beta) \in \mu^{-1}(\zeta)$ is fixed by an element $f \in F-T$. We can decompose $R$ into the eigenspace of $f$ and obtain at least two $\Gamma$-invariant parts

$$
R=R^{\prime} \oplus R^{\prime \prime}
$$

These will be preserved by $\alpha$ and $\beta$, and the pair $(\alpha, \beta)$ therefore defines an element of the quaternion module

$$
M^{\prime}=\operatorname{Hom}_{\Gamma}\left(R^{\prime}, Q \otimes R^{\prime}\right) .
$$

Denote by $F^{\prime}$ the group of those unitary transformations of $R^{\prime}$ which commute with $\Gamma$ and let $T^{\prime}$ be the scalar subgroup. We may take it that $F^{\prime} / T^{\prime}$ acts freely on $(\alpha, \beta)$, for if it did not then we could further decompose $R^{\prime}$, just as we decomposed $R$, until this condition was met. 
The condition that $F^{\prime} / T^{\prime}$ acts freely on the orbit of $(\alpha, \beta)$ means that the hyper-Kähler quotient of $M^{\prime}$ by $F^{\prime} / T^{\prime}$ is a nonsingular manifold at at least one point. From the formula for the dimension of a hyper-Kähler quotient (Proposition 2.1), we deduce the inequality $4 \operatorname{dim}_{\mathbf{R}}\left(F^{\prime} / T^{\prime}\right) \leq \operatorname{dim}_{\mathbf{R}}\left(M^{\prime}\right)$, or in other words

$$
2 \operatorname{dim}_{\mathbf{C}} \operatorname{End}_{\Gamma}\left(R^{\prime}\right)-\operatorname{dim}_{\mathbf{C}} \operatorname{Hom}_{\Gamma}\left(R^{\prime}, Q \otimes R^{\prime}\right) \leq 2 .
$$

If the decomposition of $R^{\prime}$ into irreducibles is $R^{\prime}=\bigoplus n_{i}^{\prime} R_{i}$, then (2.9) can be written

$$
2 \sum_{i}\left(n_{i}^{\prime}\right)^{2}-\sum_{i, j} a_{i j} n_{i}^{\prime} n_{j}^{\prime} \leq 2
$$

or $\sum_{i, j} c_{i j} n_{i}^{\prime} n_{j}^{\prime} \leq 2$, where $\bar{C}=\left(c_{i j}\right)$ is the extended Cartan matrix. Now let $\theta$ be defined by

$$
\theta=\sum_{0}^{r} n_{i}^{\prime} \theta_{i} .
$$

This $\theta$ is nonzero and the inequality above says that $\|\theta\|^{2} \leq 2$, where the norm is defined by the Cartan matrix. Amongst all integer linear combinations of roots, the roots themselves are characterized by just this inequality, and we conclude that $\theta$ is a root.

If $\pi: R \rightarrow R^{\prime}$ is the projection, then the element $\sqrt{-1} \pi \in f$ acts trivially on $(\alpha, \beta)$ and it follows from the formulas (2.3) for the moment maps that $\varsigma(\sqrt{-1} \pi)=0$ when we regard $\varsigma$ as a map $f \rightarrow \mathbf{R}^{3}$. By the definition of the isomorphism $\tau$, this relation means that $\varsigma \in \mathbf{R}^{3} \otimes D_{\theta}$, which is just what the proposition asserts.

Let $\left(\mathbf{R}^{3} \otimes Z\right)^{\circ}$ denote the "good" set, i.e., let

$$
\left(\mathbf{R}^{3} \otimes Z\right)^{\circ}=\left(\mathbf{R}^{3} \otimes Z\right) \backslash \bigcup_{\theta}\left(\mathbf{R}^{3} \otimes D_{\theta}\right)
$$

Corollary 2.10. If $\varsigma \in\left(\mathbf{R}^{3} \otimes Z\right)^{\circ}$, then $X_{\varsigma}$ is a nonsingular hyper-Kähler 4-manifold.

Proof. This now follows from Proposition 2.1. For the dimension of $X_{\zeta}$ we have

$$
\operatorname{dim} X_{\varsigma}=\operatorname{dim} M-4 \operatorname{dim}(F / T)=4|\Gamma|-4(|\Gamma|-1)=4
$$

\section{Properties of the manifolds}

By its definition, the regular representation has an orthonormal basis $\left\{e_{\gamma}\right\}$ indexed by $\gamma \in \Gamma$ with the property that $R(\delta) e_{\gamma}=e_{\delta \gamma}$ for all $\gamma, \delta \in \Gamma$. Let 
$L \subset M$ consist of all $(a, b) \in M$ for which $a$ and $b$ are diagonal matrices with respect to this basis of $R$. Thus if $(a, b) \in L$, then there exists, for each $\gamma \in \Gamma$, a pair $\left(a_{\gamma}, b_{\gamma}\right) \in \mathbf{C}^{2}$ such that

$$
a \cdot e_{\gamma}=a_{\gamma} e_{\gamma}, \quad b \cdot e_{\gamma}=b_{\gamma} e_{\gamma} .
$$

Because of the relations (2.2), the set of pairs $\left\{\left(a_{\gamma}, b_{\gamma}\right) \mid \gamma \in \Gamma\right\}$ must be an orbit of $\Gamma$ in $\mathbf{C}^{2}$ and we can identify $L$ with $\mathbf{C}^{2}$ by the assignment $(a, b) \mapsto$ $\left(a_{1}, b_{1}\right)$. The space $L$ then inherits from $\mathbf{C}^{2}$ an action of $\Gamma$.

Lemma 3.1. Each orbit of $F$ in $\mu^{-1}(0)$ meets $L$ in one orbit of $\Gamma$.

Proof. Take $(\alpha, \beta) \in \mu^{-1}(0)$. According to (2.3) we have $[\alpha, \beta]=0$ and $\left[\alpha, \alpha^{*}\right]+\left[\beta, \beta^{*}\right]=0$; and manipulating these two equations we obtain

$$
\left[\alpha^{*},\left[\alpha, \alpha^{*}\right]\right]+\left[\beta^{*},\left[\beta, \alpha^{*}\right]\right]=0,
$$

or $\left(A^{*} A+B^{*} B\right)\left(\alpha^{*}\right)=0$, where $A=\operatorname{ad}(\alpha)$ and $B=\operatorname{ad}(\beta)$. The positivity of $A^{*} A$ and $B^{*} B$ now implies that $A^{*} A\left(\alpha^{*}\right)=0$, and hence $\left[\alpha, \alpha^{*}\right]=\left[\beta, \beta^{*}\right]=0$. So $\alpha$ and $\beta$ are commuting normal linear transformations, and so they cannot be nilpotent unless they are zero. Let us assume that $\alpha$ and $\beta$ are not both zero.

Since they commute, $\alpha$ and $\beta$ have a simultaneous unit eigenvector $v_{1} \in R$ with

$$
\alpha \cdot v_{1}=a_{1} v_{1}, \quad \beta \cdot v_{1}=b_{1} v_{1} .
$$

Since $\alpha$ and $\beta$ are not both nilpotent, we may take it that $\left(a_{1}, b_{1}\right) \neq(0,0)$. If we define $v_{\gamma}=R(\gamma) \cdot v_{1}$, then (2.2) ensures that

$$
\alpha \cdot v_{\gamma}=a_{\gamma} v_{\gamma}, \quad \beta \cdot v_{\gamma}=b_{\gamma} v_{\gamma},
$$

where $\left\{\left(a_{\gamma}, b_{\gamma}\right) \mid \gamma \in \Gamma\right\}$ is an orbit of $\Gamma$. The points $\left(a_{\gamma}, b_{\gamma}\right) \in \mathbf{C}^{2}$ are all distinct and the vectors $v_{\gamma}$ are therefore independent and even mutually orthogonal, since $\alpha$ and $\beta$ are normal. The transformation of $R$ which sends $e_{\gamma}$ to $v_{\gamma}$ is therefore an element of $F$ which carries $(\alpha, \beta)$ into $L$. Thus each orbit of $F$ in $\mu^{-1}(0)$ meets $L$. The proof of the lemma is completed by the observation that two points of $L$ lie in the same orbit of $F$ if and only if they lie in the same orbit of $\Gamma$.

Corollary 3.2. When $\zeta=0 \in \mathbf{R}^{3} \otimes Z$, the space $X_{0}$ is isometric to $\mathrm{C}^{2} / \Gamma$.

Proof. The lemma provides a bijection $X_{0} \rightarrow L / \Gamma$. The important point is that the subspace $L \subset M$ is everywhere orthogonal to the orbits of $F$. This point is easy to verify: a tangent vector to the $F$-orbit at $(\alpha, \beta) \in L$ is a pair of matrices $([\xi, \alpha],[\xi, \beta])$ for some $\xi \in \mathfrak{f}$; these matrices are zero on the diagonal, when expressed in terms of the basis $\left\{e_{\gamma}\right\}$, and so are orthogonal to $L$. The quotient metric on $X_{0}$ at a nonsingular point is obtained from the 
orthogonal complement to the tangent space of an $F$-orbit in $\mu^{-1}(0)$; and it now follows that the bijection $X_{0} \rightarrow L / \Gamma$ is an isometry when $L$ is given the metric it inherits as a subspace of $M$, namely the Euclidean metric.

Now consider some $\varsigma$ other than 0 . Let $W_{\varsigma} \subseteq \mu^{-1}(\varsigma)$ be the union of the free orbits of $F / T$ in $\mu^{-1}(\varsigma)$ and let $U_{\varsigma}=W_{\varsigma} / F$ be the image of $W_{\varsigma}$ in the quotient $X_{\varsigma}$. By Proposition 2.1, the space $X_{\varsigma}$ is nonsingular and 4-dimensional at all points of $U_{\varsigma}$. The following lemma shows that the complement $X_{\varsigma} \backslash U_{\varsigma}$ consists of isolated singularities.

Lemma 3.3. If $\varsigma \neq 0$ and $x \in X_{\varsigma} \backslash U_{\zeta}$, then a neighborhood of $x$ in $X_{\zeta}$ is homeomorphic to a neighborhood of 0 in $\mathbf{C}^{2} / \hat{\Gamma}$, where $\hat{\Gamma} \subset \mathrm{SU}(2)$ is a group with fewer elements than $\Gamma$.

Proof. Let $m=(\alpha, \beta) \in \mu^{-1}(\zeta)$ be a representative of $x$ and let $\hat{F} \subset F$ be the stabilizer of $m$. The assumptions of the lemma mean that $\hat{F}$ is a proper subgroup of $F$ which is strictly larger than $T$. Let $V \subset T_{m} M$ be the tangent space to the $F$-orbit of $m$, and let $\hat{M}$ be the orthogonal complement in $T_{m} M$ to the $\mathbf{H}$-submodule $V+I V+J V+K V$. The space $\hat{M}$ is itself an $\mathbf{H}$-module, and the group $\hat{F}$ acts on it preserving this structure. We can therefore introduce the hyper-Kähler quotient $\hat{\mu}^{-1}(0) / \hat{F}$; we take $\hat{\mu}$ to be the unique hyper-Kähler moment map on $\hat{M}$ which vanishes at the origin. As a first step in the proof of the lemma, we shall show that a neighborhood of $x$ in $X_{S}$ is homeomorphic to a neighborhood of zero in $\hat{\mu}^{-1}(0) / \hat{F}$.

If we decompose the Lie algebra $\mathfrak{f}$ into linear subspaces $\hat{\mathfrak{f}} \oplus \hat{\mathfrak{f}}^{\perp}$, then $\hat{\mu}$ is just the component of $\mu$ in the $\hat{f}^{*}$ direction: we can write

$$
\mu(m+\varepsilon)=\varsigma+\hat{\mu}(\varepsilon)+\nu(\varepsilon)
$$

for some $\nu: \hat{M} \rightarrow \mathbf{R}^{3} \otimes\left(\hat{\mathfrak{f}}^{\perp}\right)^{*}$; here we identify $T M$ with $M$. Every $F$-orbit sufficiently close to $m$ meets $V^{\perp}$ in one orbit of $\hat{F}$; so a neighborhood of $x$ in $\mu^{-1}(\zeta) / F$ is homeomorphic to a neighborhood of $x$ in

$$
\left(V^{\perp} \cap \mu^{-1}(\varsigma)\right) / \hat{F} \text {. }
$$

Since the derivative of $\nu$ at $\varepsilon=0$ has full rank, we can replace this second space by

$$
\left(V^{\perp} \cap \hat{\mu}^{-1}(0) \cap \operatorname{Ker}(d \nu)\right) / \hat{F} .
$$

Finally, noting that $V^{\perp} \cap \operatorname{Ker}(d \nu)$ is just $\hat{M}$, we have the desired conclusion: a neighborhood of $x$ in $X_{\zeta}$ is homeomorphic to a neighborhood of 0 in $\hat{\mu}^{-1}(0) / \hat{F}$. We shall finish the proof of Lemma 3.3 by showing that $\hat{\mu}^{-1}(0) / \hat{F}$ is $\mathbf{C}^{2} / \hat{\Gamma}$ for some $\hat{\Gamma} \subset \mathrm{SU}(2)$.

Since the stabilizer of $m$ is larger than $T$, we can follow the proof of Proposition 2.8 and decompose $R$ into orthogonal $\Gamma$-invariant parts

$$
R=R^{\prime} \oplus R^{\prime} \oplus R^{\prime \prime \prime} \oplus \cdots,
$$


which are preserved by $\alpha$ and $\beta$. As was shown in Proposition 2.8, we may take it that the subgroup $\mathrm{U}\left(R^{\prime}\right) \subset \mathrm{U}(R)$ meets the stabilizer $\hat{F}$ only in the scalar subgroup $T^{\prime}$ :

$$
\mathrm{U}\left(R^{\prime}\right) \cap \hat{F}=T^{\prime} \subset \mathrm{U}\left(R^{\prime}\right)
$$

and from this follows the equality

$$
2 \operatorname{dim}_{\mathbf{C}} \operatorname{End}_{\Gamma}\left(R^{\prime}\right)-\operatorname{dim}_{\mathbf{C}} \operatorname{Hom}_{\Gamma}\left(R^{\prime}, Q \oplus R^{\prime}\right)=2 .
$$

By further decomposing $R^{\prime \prime}$ etc., we can arrange that (3.5) and (3.6) hold for all the summands in the decomposition (3.4). Define now an equivalence relation on these summands by declaring that $R^{\prime} \sim R^{\prime \prime}$ if and only if there is a $\Gamma$-invariant isometry $R^{\prime} \rightarrow R^{\prime \prime}$ which commutes with $\alpha$ and $\beta$. Such an isometry, when it exists, is unique to within a scalar multiple because of (3.5). Grouping together equivalent summands, we rewrite the decomposition (3.4) in the form

$$
R=\bigoplus_{i} \mathrm{C}^{\hat{n}_{i}} \otimes R^{(i)}
$$

where $R^{(i)} \not R^{(j)}$ unless $i=j$, and $\hat{n}_{i}$ is the number of summands equivalent to $R^{(i)}$.

We now have the following expressions for $\hat{M}$ and $\hat{F}$ :

$$
\hat{M}=\bigoplus_{i, j} \hat{a}_{i j} \operatorname{Hom}\left(\mathbf{C}^{\hat{n}_{i}}, \mathbf{C}^{\hat{n}_{j}}\right), \quad \hat{F}=\underset{i}{\times} \mathrm{U}\left(\hat{n}_{i}\right)
$$

where $\hat{a}_{i j}=\operatorname{dim}_{\mathbf{C}}\left(\hat{M} \cap \operatorname{Hom}_{\Gamma}\left(R^{(i)}, Q \otimes R^{(j)}\right)\right)$. The matrix $\hat{A}=\left(\hat{a}_{i j}\right)$ is symmetric and from (3.6) we have $\hat{a}_{i i}=0$ for all $i$. The same dimensioncounting as was used in Proposition 2.8 shows that $2 I-\hat{A}$ is positive semidefine and that the null space of $2 I-\hat{A}$ is spanned by the vector $\left(\hat{n}_{1}, \hat{n}_{2}, \cdots\right)$. This information is enough for us to conclude that $\hat{A}$ is the adjacency matrix of an extended simply-laced Dynkin diagram associated to some $\hat{\Gamma} \subset \mathrm{SU}(2)$, and the lemma now follows from Corollary 3.2, for the decompositions of $\hat{M}$ and $\hat{F}$ given in (3.7) are of just the same form as the decompositions of $M$ and $F$ given in (2.4) and (2.5).

We wish to regard the singular members of the family $X_{\varsigma}$ as singular algebraic varieties. For this purpose, let us choose just one of the complex structures, say $I$, and suppose for the moment that $N \subset M$ is any affine subvariety (with respect to $I$ ) which is invariant under $F$. In this situation there are two quotients of $N$ one can consider. First there is the affine algebro-geometric quotient $N / / F^{c}$ of $N$ by the reductive group $F^{c}$, the complexification of $F$. Secondly, there is the Kähler quotient $\left(N \cap \mu_{1}^{-1}(0)\right) / F$. The result we require is that these two are the same: the inclusion $\left(N \cap \mu_{1}^{-1}(0)\right) \rightarrow N$ and the quotient map $N \rightarrow N / / F^{c}$ together give a map $\left(N \cap \mu_{1}^{-1}(0)\right) / F \rightarrow N / / F^{c}$ which 
is a homeomorphism when $N / / F^{c}$ is given the usual (complex) topology. This result is proved in [11] for the more involved case of projective varieties. The affine case is easily deduced from the proof given there.

It is noted in [9] that if the second two components $\mu_{2}$ and $\mu_{3}$ of a hyperKähler moment map are combined into one map $\mu_{c}=\mu_{2}+i \mu_{3}$,

$$
\mu_{c}: M \rightarrow f^{*} \otimes \mathbf{C},
$$

then this complex moment map is holomorphic with respect to $I$. In our case indeed, we have $\mu_{c}(\alpha, \beta)=[\alpha, \beta]$. It follows that the level sets of $\mu_{c}$ are affine subvarieties of $M$. We deduce:

Lemma 3.9. If the first component $\varsigma_{1}$ of $\varsigma$ is zero, then $X_{\zeta}$ has the structure of an affine variety with respect to $I$.

Proof. By its definition,

$$
X_{\zeta}=\left(\mu_{1}^{-1}(0) \cap \mu_{c}^{-1}\left(\varsigma_{2}+i \zeta_{3}\right)\right) / F
$$

and by the equivalence of Kähler and algebro-geometric quotients, this is the same as $\mu_{c}^{-1}\left(\varsigma_{2}+i \varsigma_{3}\right) / / F^{c}$.

In particular, $X_{0}$ is an affine variety. The identification of $X_{0}$ with $\mathbf{C}^{2} / \Gamma$ which we made in Corollary 3.2 can be put in algebraic terms, showing that, at least if $X_{0}$ is given its reduced structure, there is an isomorphism of varieties $X_{0} \cong \mathrm{C}^{2} / \Gamma$. When $\varsigma_{1}$ is nonzero, $X_{\varsigma}$ will still be quasiprojective variety, but need not be affine.

Suppose now that $\zeta=\left(0, \varsigma_{2}, \varsigma_{3}\right)$ as in Lemma 3.9 and let $\tilde{\zeta}=\left(\varsigma_{1}, \varsigma_{2}, \varsigma_{3}\right)$, where $\zeta_{1}$ is so chosen that $\tilde{\zeta}$ does not lie in one of the subspaces $\mathbf{R}^{3} \otimes D_{\theta}$. By Corollary 2.10, the quotient $X_{\tilde{\zeta}}$ is a manifold. The inclusion $\mu^{-1}(\varsigma) \rightarrow$ $\mu_{c}^{-1}\left(\zeta_{2}+i \varsigma_{3}\right)$ and the algebro-geometric quotient map $\mu_{c}^{-1}\left(\zeta_{2}+i \zeta_{3}\right) \rightarrow X_{\zeta}$ together give a map $\lambda: X_{\tilde{s}} \rightarrow X_{\varsigma}$ which is holomorphic with respect to $I$.

Proposition 3.10. The map $\lambda: X_{\tilde{\zeta}} \rightarrow X_{\varsigma}$ is a minimal resolution of singularities.

Proof. Let us first show that $\lambda$ is proper. Let $C \subset X_{\zeta}$ be compact and let $B$ be the preimage of $\lambda^{-1}(C)$ in $\mu^{-1}(\tilde{\zeta})$. On the set $B$, the spectral radii $\sigma(\alpha)$ and $\sigma(\beta)$ are bounded, for these functions are bounded on $C$ and are constant on the orbits of the complex group $F^{c}$. The compactness of $B$ (and the properness of $\lambda$ ) therefore follows from:

Lemma 3.11. Let $B \subset M$ be a closed set on which the following functions are bounded:

(i) the spectral radii $\sigma(\alpha)$ and $\sigma(\beta)$;

(ii) the norm $|\mu|$ of the moment.

Then $B$ is compact. 
Proof. In the proof of Lemma 3.1 it was shown that if $\alpha, \beta$ were nilpotent and $\mu(\alpha, \beta)=0$, then $\alpha=\beta=0$. So the functions (i) and (ii) are simultaneously zero only at the origin. From this and the homogeneity of the two functions, the lemma follows.

Next we show that $\lambda$ is an isomorphism away from the singular set of $X_{\varsigma}$. From Corollary 3.2 we know that the nonsingular points comprise precisely the set $U_{\varsigma} \subset X_{\varsigma}$; so we must prove that if $x \in U_{\zeta}$, then $\lambda^{-1}(x)$ consists of just one point.

Let $x \in U_{\varsigma}$ and let $\pi^{-1}(x)$ be the preimage of $x$ under the algebraic quotient map $\pi: \mu_{c}^{-1}\left(\varsigma_{2}+i \varsigma_{3}\right) \rightarrow X_{\zeta}$. This fiber is invariant under $F^{c}$ and contains precisely one $F$-orbit $\Omega$ on which $\mu_{1}$ vanishes. By the general properties of algebro-geometric quotients, the $F^{c}$-orbit $F^{c} \Omega$ is closed and is contained in the closure of every $F^{c}$-orbit in $\pi^{-1}(x)$. But by definition of $U_{\zeta}$, this orbit has the maximum possible dimension, namely $\operatorname{dim}\left(F^{c} / T^{c}\right)$, and cannot therefore be contained in the closure of any other. It follows that $\pi^{-1}(x)$ consists of just the one $F^{c}$-orbit, $F^{c} \Omega$.

Set $\psi=\left|\mu_{1}-\varsigma\right|^{2}$. By Lemma 3.11, this function is proper on $F^{c} \Omega$ and therefore attains its minimum at some point $y$. Since $F / T$ acts freely on $F^{c} \Omega$, any critical point of $\psi$ is actually a zero of $\psi$ (see [11, p. 35]); so $\mu_{1}(y)=\varsigma_{1}$ and $\mu^{-1}(\tilde{\zeta}) \cap \pi^{-1}(x)$ is therefore nonempty: it consists of at least one orbit of $F$. That $\mu^{-1}(\tilde{\zeta}) \cap \pi^{-1}(x)$ consists of precisely one orbit of $G F$ follows from the results of $[10]$, and we see that $\lambda^{-1}(x)$ is a single point as required.

Now we must show that $\lambda^{-1}\left(U_{S}\right)$ is dense in $X_{\tilde{\zeta}}$. If it were not, then the inverse image of $X_{\tilde{\zeta}}$ of some singular point would contain a component of the manifold $X_{\tilde{\zeta}}$; and by the properness already proved, this component would be compact. We shall show that $X_{\tilde{\zeta}}$ has no compact component.

Set $\zeta^{\prime}=\left(\varsigma_{1}, 0, \varsigma_{3}\right)$ and consider the space $X_{\zeta^{\prime}}$ as an affine variety with respect to $J$. Without loss of generality we may assume that $X_{\varsigma^{\prime}}$ is nonsingular; see the proof of Corollary 3.12. Just as we defined the $I$-holomorphic map $\lambda: X_{\tilde{\zeta}} \rightarrow X_{\zeta}$, so too we can define a $J$-holomorphic map $\lambda^{\prime}: X_{\tilde{\zeta}} \rightarrow X_{\zeta^{\prime}}$. Like $\lambda$, the map $\lambda^{\prime}$ is an isomorphism away from the singular points, and it follows that $X_{\tilde{\zeta}}$ and $X_{\varsigma^{\prime}}$ are diffeomorphic. But being an affine variety, $X_{\zeta^{\prime}}$ can have no compact components of positive dimension. Neither, therefore, can $X_{\tilde{\zeta}}$.

We have now shown that $\lambda: X_{\tilde{\zeta}} \rightarrow X_{\zeta}$ is a resolution of singularities. The first Chern class of $X_{\tilde{\zeta}}$ is zero because $X_{\tilde{\zeta}}$ is hyper-Kähler, and this implies that $X_{\tilde{\zeta}}$ contains no exceptional curves of the first kind. The resolution is therefore minimal, and this completes the proof of Proposition 3.10.

Corollary 3.12. If $\varsigma \in\left(\mathbf{R}^{3} \otimes Z\right)^{\circ}$, then $X_{\varsigma}$ is diffeomorphic to the minimal resolution of $\mathbf{C}^{2} / \Gamma$. 
Proof. Set

$$
\zeta=\left(\varsigma_{1}, \varsigma_{2}, \varsigma_{3}\right), \quad \eta=\left(\varsigma_{1}, \varsigma_{2}, 0\right), \quad \xi=\left(\varsigma_{1}, 0,0\right) .
$$

We shall assume that $\varsigma_{1}$ does not lie in any $D_{\theta}$; this is a stronger condition than the hypothesis, but the loss of generality is not serious: by choosing a new orthonormal basis for $\mathbf{R}^{3}$, we can always arrange that $\zeta_{1}$ satisfies this condition.

Consider the four spaces $X_{\varsigma}, X_{\eta}, X_{\xi}, X_{0}$. By Corollary 2.10 and our assumption about $\zeta_{1}$, the first three are manifolds, while the fourth, by Corollary 3.2, is $\mathbf{C}^{2} / \Gamma$. By Proposition 3.10 there are three maps $\lambda, \lambda^{\prime}$ and $\lambda^{\prime \prime}$ which are holomorphic with repsect to $K, J$ and $I$ respectively:

$$
X_{\zeta} \stackrel{\lambda}{\rightarrow} X_{\eta} \stackrel{\lambda^{\prime}}{\rightarrow} X_{\zeta} \stackrel{\lambda^{\prime \prime}}{\rightarrow} X_{0} .
$$

Each of these is a minimal resolution of singularities; but since $X_{\xi}$ is already nonsingular, both $\lambda$ and $\lambda^{\prime}$ are diffeomorphisms. So $X_{\varsigma}$ is diffeomorphic to the minimal resolution of $X_{0}=\mathbf{C}^{2} / \Gamma$.

Next we compare the hyper-Kähler metric on $X_{\varsigma}$ with the Euclidean metric on $X_{0}=\mathbf{R}^{4} / \Gamma$. The composite of the three maps in (3.13) is a map $\Lambda: X_{\zeta} \rightarrow$ $\mathbf{R}^{4} / \Gamma$, which is bijective away from the singular point. Pulling back the hyper-Kähler metric on $X_{\varsigma}$ to $\mathbf{R}^{4} \backslash\{0\}$ via the composite

$$
\mathbf{R}^{4} \backslash\{0\} \rightarrow \mathbf{R}^{4} / \Gamma \stackrel{\Lambda^{-1}}{\longrightarrow} X_{\zeta}
$$

we obtain a metric $g_{5}$ on $\mathbf{R}^{4} \backslash\{0\}$. Let $\left(x_{1}, x_{2}, x_{3}, x_{4}\right)$ be standard coordinates on $\mathbf{R}^{4}$, let $\left(g_{\zeta}^{i j}\right)$ be the components of $g_{\zeta}$ in these coordinates, and let $\left(\delta^{i j}\right)$ be the Euclidean metric. Let $\Theta$ be coordinates on the unit sphere $S^{3}$, so that $(r, \Theta)$ are polar coordinates on $\mathbf{R}^{4}$. The following proposition says that $g_{\varsigma}$ is ALE.

Proposition 3.14. For any $\varsigma$, there is an expansion in powers of $r$

$$
g_{\varsigma}=\delta+\sum_{k \geq 2} h_{k}(\Theta) r^{-2 k}
$$

which may be differentiated term by term.

Proof. Consider first the dependence of $g_{\zeta}$ on $\zeta$. If one restricts $g_{\zeta}$ to the unit sphere $r=1$ then, since everything is analytic, there will be a power-series expansion in 5 :

$$
\left.g_{\zeta}\right|_{r=1}=\sum_{|\nu| \geq 0} f_{\nu} \varsigma^{\nu}
$$

where $\nu$ is a multi-index in the coordinates of $\zeta$. Now we exploit the homogeneity of the moment map, which is a quadratic function on $M$. The nonzero 
scalars $\mathbf{R}^{*}$ act on $M$ by dilatations and induce a map $\mu^{-1}(\varsigma) \rightarrow \mu^{-1}\left(t^{2} \varsigma\right)$, from which we deduce

$$
g_{\varsigma}(r, \Theta)=g_{r^{-2} \varsigma}(1, \Theta)
$$

Putting this with the power-series above, we obtain, for each $\varsigma$, a power series in $r^{-2}$ :

$$
g_{\varsigma}=\sum_{k \geq 0} h_{k}(\Theta) r^{-2 k}
$$

where $h_{k}=\sum_{|\nu|=k} f_{\nu} \varsigma^{\nu}$.

To complete the proof we must identify the first two terms $h_{k}$ : we must show that $h_{0}=\delta$ and $h_{1}=0$. The first of these two equalities is just the statement that $X_{0}$ is isometric to $\mathbf{C}^{2} / \Gamma$, and this was proved in Corollary 3.2.

To show that $h_{1}=0$ is to show that the first variation of $g_{5}$ with respect to $\varsigma$ at $\varsigma=0$ is zero. The hyper-Kähler metric $g_{\zeta}$ is entirely determined by its three Kähler forms $\omega_{i, s}(i=1,2,3)$; it will be enough therefore to show that

$$
\partial_{\mathbf{V}} \omega_{i, \varsigma}=0 \quad \text { at } \varsigma=0, i=1,2,3 \text {, }
$$

for every direction $\mathbf{V}=\left(V_{1}, V_{2}, V_{3}\right)$ in $\mathbf{R}^{3} \otimes Z$. A general formula for the variation of this 2 -form is given in [5] for the case of symplectic quotients. The argument adapts to the hyper-Kähler case, and we merely state the result. Away from the singularities, the projection $\mu^{-1}(\varsigma) \rightarrow \mu^{-1}(\varsigma) / F$ is a principal $F / T$-bundle. The horizontal distribution determined by the metric gives this bundle a connection whose curvature we denote by $\Omega_{\varsigma}$. The formula is then:

$$
\partial_{\mathbf{v}} \omega_{i \varsigma}=\left\langle V_{i}, \Omega_{\varsigma}\right\rangle \text {. }
$$

The right-hand side denotes the 2 -form obtained by pairing $V_{i} \in Z \subset(\mathfrak{f} / \mathfrak{t})^{*}$ with $\Omega \in \Lambda^{2} \otimes(\mathfrak{f} / \mathfrak{t})$.

Recall from the proof of Corollary 3.2 that $L \subset \mu^{-1}(0)$ meets all the $F$ orbits orthogonally. This means that the bundle $\mu^{-1}(0) \rightarrow \mu^{-1}(0) / F$ is flat and $\Omega_{0}=0$. So (3.15) shows that the variation is zero at $\zeta=0$, and this is what was wanted.

\section{The period map}

The exceptional set in the minimal resolution of $\mathbf{C}^{2} / \Gamma$ is a union of 2spheres whose intersection matrix is the negative of a Cartan matrix (see [17]):

$$
E=P_{1} \cup \cdots \cup P_{r}, \quad P_{i} \cdot P_{j}=-c_{i j} .
$$

The matrix $C=\left(c_{i j}\right)$ is the same Cartan matrix whose extended version features in McKay's observation. The second cohomology $H^{2}\left(X_{\zeta} ; \mathbf{R}\right)$ of each 
nonsingular quotient space can therefore be identified with $h$, the real Cartan algebra, while $\mathrm{H}_{2}\left(\mathrm{X}_{\varsigma} ; \mathbf{Z}\right)$ is the root lattice. Under this last identification, the classes $\Sigma$ with $\Sigma \cdot \Sigma=-2$ are the roots. These identifications can be made consistently for all $\zeta \in\left(\mathbf{R}^{3} \otimes Z\right)^{\circ}$ : there is no monodromy problem, since $\left(\mathbf{R}^{3} \otimes Z\right)^{\circ}$ is simply connected.

For $\varsigma \in\left(\mathbf{R}^{3} \otimes Z\right)^{\circ}$, let $\alpha_{i}(\zeta)$ denote the cohomology class of $\omega_{i}$ on $X_{\zeta}$ : these give maps

$$
\alpha_{i}:\left(\mathbf{R}^{3} \otimes Z\right)^{\circ} \rightarrow h, \quad i=1,2,3 .
$$

At the cohomology level, the formula (3.15) for the variation of $\omega_{i}$ shows [5] that there is a linear map $\sigma: Z \rightarrow h$ with $\alpha_{i}(\varsigma)=\sigma\left(\varsigma_{i}\right)$. Recall that another map $\tau: Z \rightarrow h$ was defined using McKay's observation (2.7), and that $\tau$ carries the hyperplane $D_{\theta}$ to the kernel of the root $\theta$. Since the nondegeneracy condition (*) in Theorem 1.1 just says that the $\alpha_{i}$ do not all lie in the kernel of any one root, that theorem will be completely proved if we can establish the following two properties of $\sigma$ :

Proposition 4.1. (i) The map $\sigma: Z \rightarrow h$ is a linear isomorphism.

(ii) If $\xi \in Z$ does not lie in any $D_{\theta}$, then $\sigma(\xi)$ does not lie in the kernel of any root.

Proof of (ii). Supposing $\xi$ satisfies this hypothesis, set $\zeta=(\xi, 0,0)$ and consider $X_{\varsigma}$ as a complex manifold with respect to $I$. By Proposition 3.10, this space is biholomorphic to $\widetilde{\mathrm{C}^{2} / \Gamma}$ and therefore contains holomorphic curves $P_{1}, \cdots, P_{r}$ whose homology classes form a set of simple roots. Now $\sigma(\xi)$ is the cohomology class of $\omega_{1}$ on $X$, and since a Kähler form is always positive on a holomorphic curve, we see that $\sigma(\xi)$ lies in the positive Weyl chamber with respect to this choice of simple roots. So $\sigma(\xi)$ does not lie in the kernel of any root.

The proof of (i) involves a substantial detour and occupies the rest of this section. Again fix attention on the complex structure $I$ and set

$$
N=\mu_{c}^{-1}(Z \otimes \mathbf{C}) \subset M, \quad Y=\left(N \cap \mu_{1}^{-1}(0)\right) / F .
$$

Because of the equivalence between Kähler and algebro-geometric quotients, $Y$ is an affine variety. Since the moment map is equivariant, $\mu_{c}$ descends to give a holomorphic map $\phi: Y \rightarrow Z \otimes \mathbf{C}$.

Choose a $\xi \in Z$ not lying on any $D_{\theta}$ and set

$$
\tilde{Y}=\left(N \cap \mu_{1}^{-1}(\xi)\right) / F \text {. }
$$

This space is not an affine variety, but by Proposition 2.8 and 2.1 it is a nonsingular Kähler manifold. As with $Y$, there is a holomorphic map $\tilde{\phi}: \tilde{Y} \rightarrow$ $Z \otimes \mathbf{C}$, and this fits into the following commutative diagram, in which $\lambda$ is 
defined as it was for Proposition 3.10:

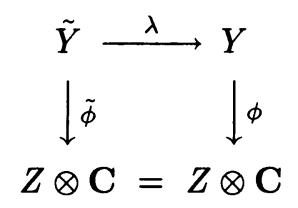

For each $\eta=\varsigma_{2}+i \varsigma_{3} \in Z \otimes \mathbf{C}$, the fiber $\phi^{-1}(\eta)$ is the affine variety $X_{\zeta}$ in the case $\varsigma=\left(0, \varsigma_{2}, \varsigma_{3}\right)$; while $\tilde{\phi}^{-1}(\eta)$ is the complex manifold $X_{\tilde{\zeta}}$, where $\tilde{\zeta}=\left(\xi, \varsigma_{2}, \varsigma_{3}\right)$. Restricted to these fibers, the map $\lambda$ is precisely the minimal resolution $\lambda: X_{\tilde{\zeta}} \rightarrow X_{\varsigma}$ of Proposition 3.10. Thus the diagram (4.2) is a simultaneous resolution of $\phi$.

Let $Y^{(n)}$ denote the normalization of $Y$. Since Lemma 3.3 shows that $Y$ is locally irreducible, the underlying topological space $\left|Y^{(n)}\right|$ is the same as $|Y|$; the two analytic spaces differ only in the local rings at their singular points, if at all.

Lemma 4.3. The map $\phi: Y^{(n)} \rightarrow Z \otimes \mathbf{C}$ is a flat deformation of $\mathbf{C}^{2} / \Gamma$.

Proof. The fibers of $\tilde{\phi}$ are a smooth family of complex surfaces in which the special fiber $\tilde{\phi}^{-1}(0)$ is isomorphic to the minimal resolution of $\mathbf{C}^{2} / \Gamma$. According to [16], such a family can be blown down fiberwise to produce a flat deformation $\check{\phi}: \check{Y} \rightarrow Z \otimes \mathbf{C}$ of $\mathbf{C}^{2} / \Gamma$; the ring $H^{0}(\check{Y} ; \mathscr{O})$ is isomorphic to $H^{0}(\tilde{Y}, \mathscr{O})$ and there is therefore a diagram:

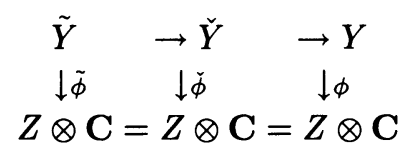

Since $\lambda: \tilde{Y} \rightarrow Y$ is proper and birational, the same is true of the map $\check{Y} \rightarrow Y$. This map is also finite, and since $\check{Y}$ is necessarily normal, it follows that $\check{Y}$ is the normalization of $Y$.

Remark. The author has no evidence against the conjecture that $Y$ is itself normal and that $\phi: Y \rightarrow Z \otimes \mathbf{C}$ is flat. It is only for lack of a direct proof of this flatness that the results of [16] are needed.

On any hyper-Kähler manifold, the complex-valued 2-form $\omega_{c}=\omega_{2}+i \omega_{3}$ is nondegenerate and holomorphic with respect to $I$ (see [9]). So away from the singular locus, $\omega_{c}$ gives a holomorphic 2-form on all the fibers $\phi^{-1}(\eta)$ of $\phi$, depending holomorphically on $\eta \in Z \otimes \mathbf{C}$. In the sense of [14], the map

$$
\sigma \otimes 1: Z \otimes \mathbf{C} \rightarrow h \otimes \mathbf{C}
$$

is therefore the period map for this deformation of $\mathbf{C}^{2} / \Gamma$. 
Let $\Psi: \mathscr{Y} \rightarrow \mathscr{V}$ be the semi-universal deformation of $\mathbf{C}^{2} / \Gamma$ and let $s: Z \otimes$ $\mathrm{C} \rightarrow \mathscr{V}$ be the map by which $\phi$ is induced from $\Psi$ :

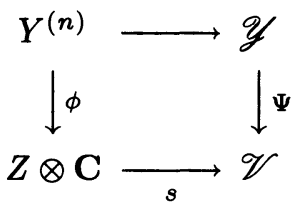

The space $Y^{(n)}$ inherits from $M$ an action of the scalars $\mathbf{C}^{*}$, and since $\mu^{c}$ is quadratic, the map $\phi$ will be equivariant if we make $\mathbf{C}^{*}$ act on $Z \otimes \mathbf{C}$ with weight 2 . Thus $\phi$ is a $\mathbf{C}^{*}$-deformation, and if we take $\Psi$ to be the $\mathbf{C}^{*}$-semiuniversal deformation (see [17]), then it follows that we may take it that $s$ is $\mathbf{C}^{*}$-equivariant and globally defined.

At this point we need a result due to Looijenga [14] which implies that a deformation such as $\phi$ is entirely determined by its period map. We shall go into this a little more carefully than our present situation requires, for we will have need of the result again in [13].

The first thing is that $\Psi$ admits a simultaneous resolution; this implies, in particular, that the minimal resolution of every fiber is diffeomorphic to $\widetilde{\mathbf{C}^{2}} / \Gamma$. The construction of this simultaneous resolution is due to Brieskorn [1], [2], [3], Slodowy [17], and independently to Tjurina [19]; it is a corollary of Brieskorn's description that the base $\mathscr{V}$ of $\Psi$ is naturally identified with $(h \otimes \mathbf{C}) / W$. Under this identification the discriminant locus $\mathscr{D} \subset \mathscr{V}$, i.e., the set of $v \in \mathscr{V}$ for which $\Psi^{-1}(v)$ is singular, is carried onto the branch locus of the quotient map $h \otimes \mathbf{C} \rightarrow(h \otimes \mathbf{C}) / W$, the projection of the kernels of the roots. Choosing a base-point $v_{0} \in \mathscr{V} \backslash \mathscr{D}$, one obtains a natural monodromy representation on the second cohomology [14],

$$
\pi_{1}(\mathscr{V} \backslash \mathscr{D}) \rightarrow \operatorname{Aut}\left(H^{2}\left(\Psi^{-1}\left(v_{0}\right) ; \mathbf{C}\right)\right) .
$$

This representation was calculated in [18] and shown to coincide with the standard representation of $W$ on $h \otimes \mathbf{C}$.

Away from the singular points, the fibers of $\Psi$ carry a holomorphic 2-form depending holomorphically on the base, and one therefore has a period map $p_{\Psi}$; because of the monodromy, it takes values in $(h \otimes \mathbf{C}) / W$ :

$$
p_{\Psi}: \mathscr{V} \backslash \mathscr{D} \rightarrow(h \otimes \mathbf{C}) / W
$$

Looijenga's result is that $p_{\Psi}$ extends across $\mathscr{D}$ and coincides with the standard isomorphism between $\mathscr{V}$ and $(h \otimes \mathbf{C}) / W$. From this one may deduce the following.

Proposition 4.5. Let $\phi: Y \rightarrow V$ be a $\mathbf{C}^{*}$-deformation of $\mathbf{C}^{2} / \Gamma$ whose generic fiber is nonsingular, and suppose that the only $\mathbf{C}^{*}$-invariant neighborhood of the distinguished point in $Y$ is $Y$ itself. Then $\phi$ is determined 
by its period map $p$ : precisely, $\phi$ is the pull-back of $\Psi$ via the composite $\left(p_{\Psi}\right)^{-1} \circ p: V \rightarrow \mathscr{V}$.

Proof. The hypotheses ensure that $\phi$ is the pull-back of $\Psi$ by some homogeneous map $s: V \rightarrow \mathscr{V}$. The pull-back of the 2-form of the fibers of $\Psi$ gives a 2 -form on the fibers of $\phi$, and in the presence of the $\mathbf{C}^{*}$-action, this object is essentially unique. It follows that the period map for $\phi$ is the composite of $s$ and $p_{\Psi}$. This proves the proposition.

We can now prove Proposition 4.1(i). From Lemma 3.3 we see that $\phi^{-1}(0)$ is the only fiber of $\phi$ which is isomorphic to $\mathbf{C}^{2} / \Gamma$, and from this it follows that, in the diagram (4.4), we have $s^{-1}(0)=\{0\}$. Proposition 4.5 then implies that the period map has the same property, that is $\sigma^{-1}(0)=\{0\}$. Since $\sigma$ is a linear map between spaces of equal dimension, it must be an isomorphism.

Remark. Having now calculated the period map of $\phi$, we see that this deformation is obtained from the semi-universal deformation by lifting through the Weyl group. The diagram (4.2) is therefore the simultaneous resolution of the semi-universal deformation constructed by Brieskorn, Slodowy and Tjurina.

Acknowledgements. This work formed part of the author's doctoral thesis and was prepared under the supervision of M. F. Atiyah, to whom the author is particularly grateful for all the help and advice he has received. The author also wishes to thank N. J. Hitchin and P. Slodowy for answering many questions in the course of several much-appreciated conversations.

\section{References}

[1] E. Brieskorn, Über die Auflösung gewisser Singularitäten von holomorphen Abbildungen, Math. Ann. 166 (1966) 76-102.

[2] _ Die Auflösung der rationalen Singularitäten holomorpher Abbildungen, Math. Ann. 178 (1968) 244-270.

[3] _ Singular elements of semisimple algebraic groups, Actes Congr. Internat. Math., No. 2, 1970, 279-284.

[4] E. Calabi, Métriques kahlériennes et fibrés holomorphes, Ann. Sci. École. Norm. Sup. (4) 12 (1979) 269-294.

[5] J. J. Duistermaat \& G. J. Heckman, On the variation in the cohomology of the symplectic form in the reduced phase space, Invent. Math. 69 (1982) 259-268.

[6] T. Eguchi, P. B. Gilkey \& A. J. Hanson, Gravitation, gauge theories and differential geometry, Phys. Rev. 66 (1980) 215-393.

[7] G. W. Gibbons \& S. W. Hawking, Gravitational multi-instantons, Phys. Lett. B 78 (1978) 430-432.

[8] N. J. Hitchin, Polygons and gravitons, Math. Proc. Cambridge Philos. Soc. 83 (1969) 465-476.

[9] N. J. Hitchin, A. Karlhede, U. Lindström \& M. Roðek, Hyperkähler metrics and supersymmetry, Comm. Math. Phys. 108 (1987) 535-589.

[10] G. Kempf \& L. Ness, The length of vectors in representation spaces, Algebraic Geometry, Lecture Notes in Math., Vol 732 (K. Lønsted, ed.), Springer, Berlin, 1978, 233-242. 
[11] F. C. Kirwan, Cohomology of quotients in symplectic and algebraic geometry, Math. Notes 31, Princeton University, 1984.

[12] P. B. Kronheimer, Instantons gravitationnels et singularités de Klein, C. R. Acad. Sci. Paris Sér. I Math. 303 (1986) 53-55.,

[13] _ , A Torelli-type theorem for gravitational instantons, J. Differential Geometry 29 (1989), 685-697.

[14] E. J. N. Looijenga, Isolated singular points on complete intersections, London Math. Soc. Lecture Note Series 77, Cambridge University Press, 1984.

[15] J. McKay, Graphs, singularities and finite groups, Proc. Sympos. Pure Math. Vol. 37, Amer. Math. Soc., 1980, 183-186.

[16] O. Riemenschneider, Familien komplexer Räume mit streng pseudokonvexer spezieller Faser, Comment. Math. Helv. 51 (1976) 547-565.

[17] P. Slodowy, Simple singularities and simple algebraic groups, Lecture Notes in Math., Vol. 815, Springer, Berlin, 1980.

[18] _ Four lectures on simple groups and singularities Commun. Math. Inst. Rijksuniv. Utrecht 11 (1980).

[19] G. N. Tjurina, Resolutions of singularities of flat deformations of rational double points, Functional Anal. Appl. 4 (1970) 68-73.

INSTITUTE FOR ADVANCED STUDY 
